Expanding the bandwidth of rotational accelerometers by autonomous gain-phase tailoring

This article has been downloaded from IOPscience. Please scroll down to see the full text article.

2003 Smart Mater. Struct. 12210

(http://iopscience.iop.org/0964-1726/12/2/308)

The Table of Contents and more related content is available

Download details:

IP Address: 140.112.113.225

The article was downloaded on 25/12/2008 at 02:16

Please note that terms and conditions apply. 


\title{
Expanding the bandwidth of rotational accelerometers by autonomous gain-phase tailoring
}

\author{
Yu-Hsiang Hsu and Chih-Kung Lee \\ Institute of Applied Mechanics, National Taiwan University, Taipei, Taiwan \\ Received 21 May 2002, in final form 18 November 2002 \\ Published 20 February 2003 \\ Online at stacks.iop.org/SMS/12/210
}

\begin{abstract}
The concept of an autonomous gain-phase piezoelectric device is adapted to the design of a rotational accelerometer. It can be shown that a no-phase-delay low-pass filter can be introduced into the transfer function of an accelerometer by weighting the structure vibration strain spatially. This can be achieved by modifying either the shape or the effective surface electrode of a thin layer piezoelectric film. With the introduction of this new class of filter, the usable bandwidth of the rotational accelerometer created can be shown to expand significantly. It can also be demonstrated that the concept is applicable to mechanical systems that can be represented using a second-order partial differential equation including a shaft, rod, etc. To verify the performance of the newly developed rotational accelerometer, which is difficult to test due to its wide bandwidth, the design and implementation of a testing methodology based on a wideband rotational shaker is also detailed.
\end{abstract}

\section{Introduction}

During the last two decades, studies on the placement of distributed sensors and actuators on structures to enhance overall performance have been actively undertaken [1-4]. Following this line of thinking and then implementing the results for distributed sensors/actuators in the development of point sensors, a whole new technology that provides us with new capabilities for tailoring system performance with autonomous gain and phase characteristics has been developed [5]. This new class of sensors has been named APROPOS (autonomous phase-gain rotation/linear piezoelectric optimal sensing) [5]. The concept relating to an APROPOS device clearly demonstrates that the design concept of distributed sensors $[6,7]$ can be successfully merged with that of point sensors to form point-distributed sensors (PoD sensors) [5]. One of the main advantages of this type of sensor is its ability to extend the useful bandwidth of the overall sensor beyond the limitations imposed by the sensor structure itself. Previously reported APROPOS devices were developed on flexible bending structures such as beams or one-dimensional plates, both of which have fourth-order characteristic polynomials. A total of four wave modes, two propagating and two evanescent waves, are present in these sensor structures. In this paper, we show that rotational type APROPOS devices are possible as well. It should be noted that two propagating waves instead of four wave modes are present in the sensor structure under torque. It will also be shown that this fundamental difference will have a large impact on the design of the rotational type APROPOS device. Both the design concept and the implementation of the newly developed rotational APROPOS device will be discussed in this paper. Due to the design innovation of using an APROPOS device in point rotational sensors, the operational bandwidth can be easily expanded to the kilohertz range. To verify the performance of this type of rotational APROPOS device, the bandwidth of the rotational shakers used must also be expanded. The methodologies developed to expand the testing bandwidth of traditional rotational shakers are also presented. It will be shown in this paper that by using a matching filter [8], which has been well developed in high-performance audio systems, a wideband rotational shaker can be made by placing a servomotor and a piezoelectric rotational shaker in tandem. In summary, the theory, the design of the experimental set-up and the experimental results will all be detailed in this paper. 


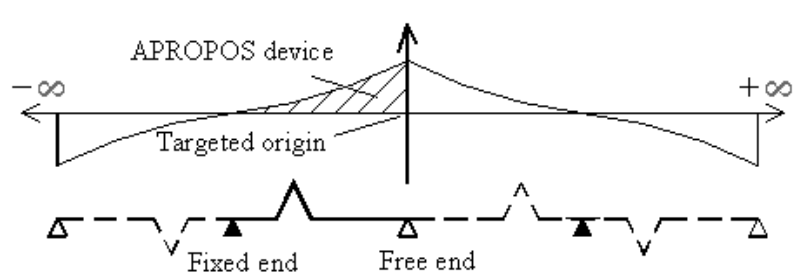

Figure 1. Schematic of a wave mode and an APROPOS device in an infinite domain.

\section{Concept of an APROPOS device}

The fundamental principle of an APROPOS device is based on consideration of a methodology that controls the spatial weighting factors of the wave modes of a structure with a shaped piezoelectric thin film mounted on top of a onedimensional cantilever plate [5]. The APROPOS device can be implemented by the following two methods. The first method is the method of images [9], which maps the wave modes on the finite domain to an infinite domain by considering the characteristics of the boundary such as that of fixed or free ends [5]. The second method involves adopting window functions to eliminate the effect of the waves outside the finite domain [10], i.e. making sure that the weighting factor for the boundary is zero [5]. It should be noted that the method of integrating the window function concept to tailor a structural system has been well developed in the area of surface acoustic devices (SAW devices) [11, 12]. Both methods can expand the wave mode propagation between the boundaries of the finite structure into the infinite domain. That is, the combined effect of the APROPOS device and the wave modes in the structure is continuous in the infinite domain. Figure 1 shows further details of this characteristic. The waves, which propagate to and from the fixed end, will have a $180^{\circ}$ phase delay. However, the waves propagating to and from the free end will not induce any phase delay. More specifically, the waves will be continuous for the free end but will show a jump at the fixed end. By introducing the concept of an APROPOS device, the discontinuity can be eliminated by making sure that the sensor has zero weighting at the fixed end, as is shown in figure 1. In other words, an APROPOS device actually specifies the spatial weighting factors for the wave modes, which can also be understood by saying that wave modes keep on 'seeing' an APROPOS device as they propagate between the boundaries. With either of the two methods discussed above, the waves on the finite structure can be perceived as propagating continuously in a mapped infinite domain. That is, a domain from minus infinity to infinity is constructed spatially using the two above-mentioned methods. It is then not surprising to realize that a no-phase-delay filter can be implemented, as information about the past and future of the structure exists simultaneously, represented by the right and left propagating waves, while integrating the contribution of the signal within the APROPOS device [5, 7].

\subsection{Rotational APROPOS device based accelerometers}

Compared with the equation governing the bending of a onedimensional plate, which is a fourth-order partial differential equation (PDE), the equation governing the torsional vibration of a shaft is a second-order PDE. More specifically, the equation governing the torsional vibration of a shaft can be written as [9]:

$$
G \frac{\partial^{2} \theta(x, t)}{\partial x^{2}}-\rho \frac{\partial^{2} \theta(x, t)}{\partial t^{2}}=0
$$

where the constant $G$ is the shear modulus, $\rho$ is the density, $x$ is the spatial coordinate, $t$ is time and $\theta(x, t)$ is the twisting angle. Note that the damping effect is neglected in equation (1). Considering only the harmonic structure response, i.e. $\theta(x, t)=\theta(x) \mathrm{e}^{\mathrm{j} \omega t}$ and $\mathrm{j}=(-1)^{1 / 2}$ leads to

$$
\frac{\partial^{2} \theta(x)}{\partial x^{2}}+k^{2} \theta(x)=0,
$$

where

$$
k^{2}=-\frac{\rho}{G} \omega^{2}
$$

is the dispersion relationship between the wavenumber $k$ and frequency $\omega$. Thus the general solution of the one-dimensional shaft with a solid circular cross section can be expressed as two propagating waves, i.e. the right and the left propagating wave modes in equation (4):

$$
\theta(x, t)=\left[w_{l p} \mathrm{e}^{\mathrm{j} k x}+w_{r p} \mathrm{e}^{-\mathrm{j} k x}\right] \mathrm{e}^{\mathrm{j} w t}
$$

where $w_{l p}$ and $w_{r p}$ are the wave mode amplitudes of the left and the right propagating waves and are functions of wavenumber $k$ only. It should be noted that these wave mode amplitudes really represent the fundamental characteristics of the structure, which are determined by the boundary conditions of the structure. That is, the boundary condition determines the characteristic of the sensor structure and thus also the overall performance of the rotational accelerometer. As the sensor characteristic is highly dependent on the characteristics of the sensor structure chosen, any methodology that can be used to reduce the overall dependency of the sensor response to that of the sensor structure itself will be highly valuable in tuning the overall sensor response. Therefore, introducing an APROPOS device to create a no-phase-delay low-pass filter to improve the sensor frequency response by extending the usable bandwidth will be invaluable. It should be noted that this series of spatial filters is fundamentally different from traditional electric filters, as a phase delay will inevitably be introduced into the sensor frequency whenever a traditional electric filter is introduced, as per the well-known Bode gainphase theorem [13].

The rotational APROPOS device based accelerometer and its effect is shown in figure 2, where $G_{o}(s)$ is the original transfer function of the sensor structure, $G_{s}(s)$ is the sensor transfer function that has been tailored using the APROPOS device concept, $F_{l}(s)$ is the transfer function of a low-pass filter, $\mathrm{BW}_{o}$ is the original bandwidth of the sensor structure, $\mathrm{BW}_{s}$ is the enhanced bandwidth of the sensor with the implementation of the APROPOS device concept, and $\mathrm{BW}_{E}$ is the bandwidth if an electrical filter is applied. Note that a low-pass filter implemented using the APROPOS device will induce only the gain tailoring without any phase lag, such that it significantly extends the usable bandwidth of the accelerometer. In comparison, if an electrical low-pass filter is applied, the phase will be significantly altered and no 


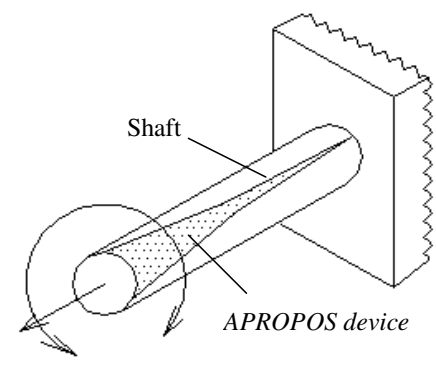

(a)

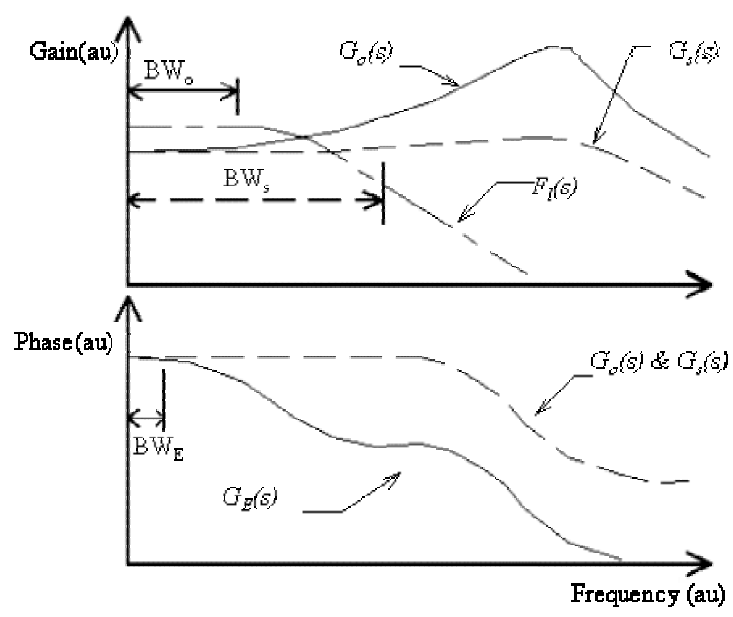

(b)

Figure 2. (a) Schematic of a rotational APROPOS device based accelerometer. (b) Schematic of the effect of an APROPOS device.

significant improvement in bandwidth will be found. Thus the implementation of an APROPOS device in the design of a rotational accelerometer is the only way to enhance the usable bandwidth of the sensor, which up to now has not been possible using electrical filters.

\subsection{Theory of piezoelectric laminates}

Piezoelectric material has been studied extensively over the last 100 years. In addition, piezoelectric laminates have also been used extensively for the last 20 years due to the demand for flexible structures. Coupling mechanical and electrical fields using the piezoelectric effect leads to the following governing equations [14]:

$$
S_{p}=s_{p q}^{E} T_{q}-d_{i p} E_{i}, \quad D_{i}=d_{i p} T_{q}+\varepsilon_{i j}^{T} E_{k},
$$

where $i, k=1-3, p, q=1-6, s_{p q}^{E}$ are the elastic compliance constants, $\varepsilon_{i j}^{T}$ and $d_{i p}$ are the permittivity and piezoelectric strain/charge constants $T_{q}, S_{p}$ are stress and strain and $D_{i}$ and $E_{k}$ represent electric displacement and electrical field respectively. It is obvious from the governing equation that as an electric field is applied, a mechanical strain will occur [14]. On the other hand, when a force is applied to the material, a charge can be measured at the surface of the material. As piezoelectric materials in thin sheet form, such as PVDF (polyvinylidene fluoride) film are used, a plane stress approximation was used to model the thin piezoelectric lamina. That is, equation (5) can be modified to $D_{3}=\varepsilon_{33} E_{3}+d_{31} T_{1}+d_{32} T_{2}$ and the induced charge can only be detected along the thickness direction. In addition, uniform poling strength throughout the thin film was assumed. It should be indicated that this equation is based on the case where the principal axes of the material and structure coincide with each other. Considering the effect of the skew angle $\theta_{s}[6,15,16]$, which refers to the angle between the principal axes of the piezoelectric material and that of the structure, this equation can be further modified to:

$$
D_{3^{\prime}}=\varepsilon_{3^{\prime} 3^{\prime}} E_{3^{\prime}}+d_{3^{\prime} 1^{\prime}} T_{1^{\prime}}+d_{3^{\prime} 2^{\prime}} T_{2^{\prime}}+d_{3^{\prime} 6^{\prime}} T_{6^{\prime}},
$$

where the primes represent the constants as referred to by the coordinate axes of the material, which are related to the principal axes of the structure by a skew angle $\theta_{s}$. It is to be noted that an additional piezoelectric constant $d_{3^{\prime} 6^{\prime}}$ appears since the skew angle is not equal to zero. Using Gauss' law to find the signals detectable by surface electrode $S^{(12)}$, which is represented by $F(x, y)$, the charge signals generated by the $k$ th lamina become $[6,14,15]$ :

$$
\begin{aligned}
q(t) & =\iint_{S^{(12)}} F(x, y)\left[e_{3^{\prime} 1^{\prime}} \frac{\partial u}{\partial x}+e_{3^{\prime} 2^{\prime}} \frac{\partial v}{\partial y}\right. \\
& \left.+e_{3^{\prime} 6^{\prime}}\left(\frac{\partial u}{\partial y}+\frac{\partial v}{\partial x}\right)\right] \mathrm{d} x \mathrm{~d} y .
\end{aligned}
$$

If the vibration of the attached structure is purely shear strain, the piezoelectric thin film is attached to the surface of the one-dimensional circular shaft. Then the shear strain can be expressed as $\varepsilon_{\theta z}=r \mathrm{~d} \theta / \mathrm{d} x$ only. Substituting equation (4) into (7), the sensor equation can be simplified as:

$$
q_{s}(k)=\mathrm{j} k e_{3^{\prime} 6^{\prime}} r^{2} \int_{0}^{a} R(x)\left[w_{l p} \mathrm{e}^{\mathrm{j} k x}-w_{r p} \mathrm{e}^{-\mathrm{j} k x}\right] \mathrm{d} x
$$

where

$$
R(x)=\int_{-b / 2}^{b / 2} F(x, y) \mathrm{d} y
$$

is the effective surface electrode of the piezoelectric thin film. One of the fundamental drivers behind the design of the reported distributed sensors is the ability to choose a proper effective surface electric function $R(x)[15,16]$. In addition, the APROPOS device also uses the same idea by weighting the wave modes in the infinite domain expanded by using either the method of images or window functions. The key principle behind an APROPOS device lies in regarding each term of equation (8) as a two-sided Laplace transform [17]. With this observation, the Laplace transform table can be used as a powerful mathematical tool for the design of APROPOS devices. One thing that should be mentioned here is that PVDF thin film does not have a $d_{3^{\prime} 6^{\prime}}$ constant. The lack of a $d_{3^{\prime} 6^{\prime}}$ constant can be compensated for by choosing a proper polarization profile or by selecting an appropriate PVDF skew angle. As the shear strain can be measured by the induced piezoelectric constant $d_{3^{\prime} 6^{\prime}}$, the existence of $d_{3^{\prime} 1^{\prime}}$ and $d_{3^{\prime} 2^{\prime}}$ will also measure the normal strain along the principal axes of the material along the $x_{1^{\prime}}$ and $x_{2^{\prime}}$ axes. Thus the charges generated on the electrodes are contributed by the piezoelectric effects through the constants $d_{3^{\prime} 1^{\prime}}, d_{3^{\prime} 2^{\prime}}$ and $d_{3^{\prime} 6^{\prime}}$. To implement a pure shear sensor, the strain that is measured by the $d_{3^{\prime} 1^{\prime}}$ and $d_{3^{\prime} 2^{\prime}}$ piezoelectric constants must be eliminated. It is 
Expanding the bandwidth of rotational accelerometers by autonomous gain-phase tailoring

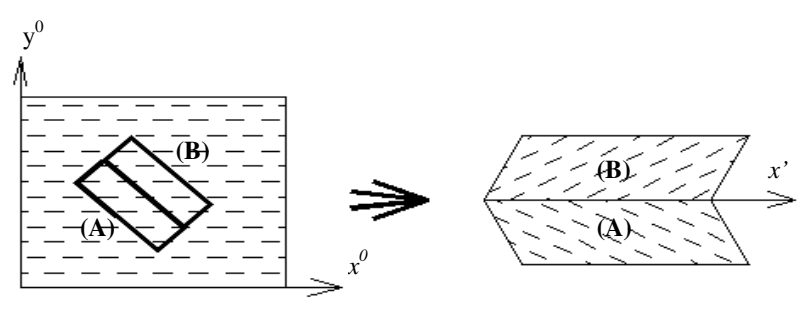

Figure 3. Schematic of a pure shear strain sensor.

known that the $d_{3^{\prime} 1^{\prime}}\left(\theta_{s}\right)$ and $d_{3^{\prime} 2^{\prime}}\left(\theta_{s}\right)$ constants of the PVDF at skew angles $\theta_{s}$ equal to $\pm \pi / 4$ are the same, and there is the difference of a minus between the $d_{36}$ constant at $\theta_{s}= \pm \pi / 4$, i.e. $d_{3^{\prime} 1^{\prime}}(\pi / 4)=d_{3^{\prime} 2^{\prime}}(\pi / 4)=d_{3^{\prime} 1^{\prime}}(3 \pi / 4)=d_{3^{\prime} 2^{\prime}}(3 \pi / 4)$, $d_{3^{\prime} 6^{\prime}}(\pi / 4)=-d_{3^{\prime} 6^{\prime}}(3 \pi / 4)[6,16]$. It is clear therefore that a pure shear sensor can be easily implemented by using the configuration shown in figure 3 . More specifically, a reversed PVDF thin film with skew angle equal to $3 \pi / 4$ on another one with skew angle equal to $\pi / 4$ will offer an additional minus sign, since the poling direction is opposite. In this way the strains that are measured by the $d_{3^{\prime} 1^{\prime}}$ and $d_{3^{\prime} 2^{\prime}}$ piezoelectric constants cancel out from these two opposite PVDF thin films. In addition, since there exists a minus sign between the $d_{3^{\prime} 6^{\prime}}$ piezoelectric constants for skew angles equal to $\pi / 4$ and $3 \pi / 4$, the output charges will be directly proportional to twice the shear strain, which is a kind of pure shear strain sensor. Note that EMI (electromagnetic interference) shielding is automatically obtained by connecting the external electrode of the folded PVDF thin film to ground.

\subsection{Implementation of rotational APROPOS devices}

Considering again the conceptual drawing shown in figure 1, it is clear that an APROPOS device is symmetric with respect to the targeted origin in the infinite domain, where the targeted origin is determined by the chosen origin. This characteristic determines the form of the wave mode amplitudes in equation (4), in which the local characteristic of the targeted origin is embedded. With this symmetry, the wave modes propagating to and from the targeted origin remain the same. The sensor equation can thus be reduced to

$$
q_{s}(k)=\mathrm{j} k e_{36} r^{2}\left(w_{l p}-w_{r p}\right) \int_{-\infty}^{\infty} R(x) \mathrm{e}^{-\mathrm{j} k x} \mathrm{~d} x .
$$

A no-phase-delay filter can then be implemented by choosing an appropriate effective surface electrode $R(x)$. It should be emphasized that the characteristic of the rotational APROPOS device based accelerometer is the superposition of (1) all characteristics of the sensor structure, (2) its local performance, and (3) the filtering effect induced by using the APROPOS device concept. Note that the APROPOS device is implemented by the effective surface electrode $R(x)$, which forms as a weighting function to the right and left propagating waves. The characteristic of the structure embedded in wave mode amplitudes will not be influenced by an APROPOS device as such a device only offers a spatial filtering effect superimposed on the frequency response of the final system.

Considering the Laplace transformed pairs listed in table 1 , it can be seen that a $-40 \mathrm{~dB} /$ decade no-phase-delay
Table 1. (a) Right-sided Laplace transformed pair where $F(s)=\int_{0}^{\infty} f(x) \mathrm{e}^{-s x} \mathrm{~d} x$. (b) Left-sided Laplace transformed pair where $F(s)=\int_{-\infty}^{0} f(x) \mathrm{e}^{-s x} \mathrm{~d} x$.

\begin{tabular}{|c|c|c|c|}
\hline \multicolumn{2}{|r|}{ (a) } & \multicolumn{2}{|r|}{ (b) } \\
\hline$f(x)$ & $F(s)=£\{f(x)\}$ & $f(x)$ & $F(s)=£\{f(x)\}$ \\
\hline \multirow{2}{*}{$\mathrm{e}^{-\alpha x}$} & 1 & \multirow{2}{*}{$\mathrm{e}^{\alpha x}$} & 1 \\
\hline & $\alpha+s$ & & $\alpha-s$ \\
\hline \multirow{2}{*}{$\sin (\gamma x)$} & $\gamma$ & \multirow{2}{*}{$\sin (\gamma x)$} & $\gamma$ \\
\hline & $s^{2}+\gamma^{2}$ & & $s^{2}+\gamma^{2}$ \\
\hline
\end{tabular}

low-pass filter can be introduced by introducing $\mathrm{e}^{-\alpha|x|}$ to the effective surface electrode. Another thing that should be noted is that an effective surface electrode with the shape of a sine function will produce a null output. In addition, the wave modes at the fixed end can be represented by odd functions and the wave modes at the free end can be represented by even functions, as described above. Choosing the fixed end as the targeted origin and the corner wavenumber $\gamma$ of the sine function at $(2 n+1) \pi / 2$, a continuous sine function can then be imaged into the infinite domain. We can then choose an effective surface electrode as [6]:

$$
R(x)=\mathrm{e}^{-\alpha x}-\mathrm{e}^{-\beta x}-c \sin [\gamma(x)]
$$

from 0 to $a$. The sine function is introduced to compensate for the effect where the exponential function approaches zero only in the infinite domain, which means that the shape of the electrode must be reduced to zero at the boundary. By using the data shown in table 1, it can be seen that the unfinished profile can be reduced to zero in the finite domain by using a sine function as the sine function integrates toward zero in the twosided Laplace transform. With equation (11), the characteristic of the rotational APROPOS device can be expressed as:

$q(k)=\mathrm{j} k e_{36} r^{2}\left(w_{l p}-w_{r p}\right)(G / \rho)^{1 / 2}\left[\frac{\left(\bar{\beta}^{2}-\bar{\alpha}^{2}\right) \mathrm{j} \omega}{\left(\bar{\alpha}^{2}+\omega^{2}\right)\left(\bar{\beta}^{2}+\omega^{2}\right)}\right]$,

where the dispersion relationship is substituted to transfer the rotational accelerometer response to the frequency domain. Note that a $-60 \mathrm{~dB} /$ decade no-phase-delay low-pass filter can be created by the APROPOS device designed here. It should be noted that equation (12) is also a filter that can be used to tailor sensor dc gain by merely choosing the proper corner wavenumber $\alpha$ or $\beta$. Note that the zero $\mathrm{j} \omega$ in the numerator is induced by the odd function of the fixed end. With the implementation of this $-60 \mathrm{~dB} /$ decade no-phasedelay low-pass filter, the usable bandwidth of the sensor can easily be expanded to $80-90 \%$ of the first mode frequency by the APROPOS device, which is significant as traditional sensors can only be used below $10 \%$ of the first mode frequency if $5 \%$ linearity is expected.

\section{Developing testing methodology: a new shaker}

With the reduction in size of the testing structure, its operational bandwidth can be extended to the kilohertz range. As traditional rotational shakers made by servomotors do not have enough bandwidth to verify the performance of the sensor, a new shaker was developed to facilitate the testing 


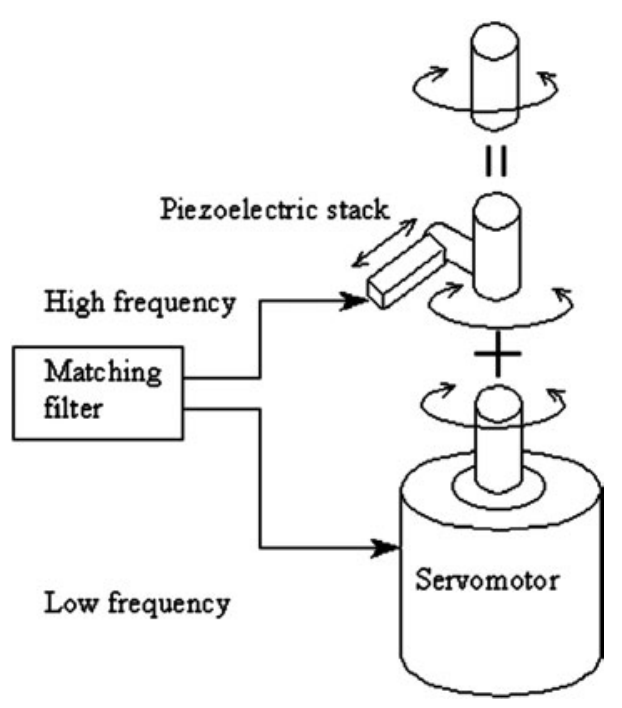

Figure 4. Schematic of the newly developed wideband rotational shaker to verify the performance of a rotational APROPOS device.

of the APROPOS device based sensor. More specifically, a servomotor can only introduce a small loading at high frequency while creating a larger loading at low frequency. Generally speaking, the usable bandwidth of a servomotor is typically lower than a few hundred hertz. To test the newly developed sensor up to the kilohertz range, a new shaker was developed which will be detailed here.

The concept of a wideband rotational shaker is realized by embedding a piezoelectric stack in the servomotor to induce high-frequency loading when the servomotor is out of range. That is, making the servomotor and the piezoelectric stack responsible for low- and high-frequency ranges respectively creates a new shaker. A matching filter [8] was used to transmit the low-frequency signal to the servomotor and the high-frequency signal to the piezoelectric stack (figure 4). As a servomotor is proficient at inducing low-frequency rotations and a piezoelectric shaker is effective in a high-frequency range, a wideband, flat frequency response can be achieved at the newly developed tandem shaker by tuning the transfer function of the matching filter. It was found that the usable bandwidth created by the rotational shaker is expanded up to $10 \mathrm{kHz}$ or even higher depending on the performance of the piezoelectric stack.

\section{Experimental set-up}

To verify the effect of the rotational APROPOS device, a $50 \mathrm{~mm}$ long, $10 \mathrm{~mm}$ diameter wide silica gel of a onedimensional shaft was chosen as the sensor structure. The experimental set-up is shown in figure 5. The characteristic of the shaft was measured by placing a uniform sensor to arrive at the frequency response curve shown in figure 6 as a thin curve. It is obvious from the experimental results that the natural frequency of the shaft is located from between several hundred hertz to $1 \mathrm{kHz}$, which indicates the necessity for a wideband rotational testing base. Considering figure 6 , the damping effect of the first mode around $70 \mathrm{~Hz}$ is large, which corresponds to the shaft structure having a low quality factor. The design parameters for an APROPOS device based filter are now presented.

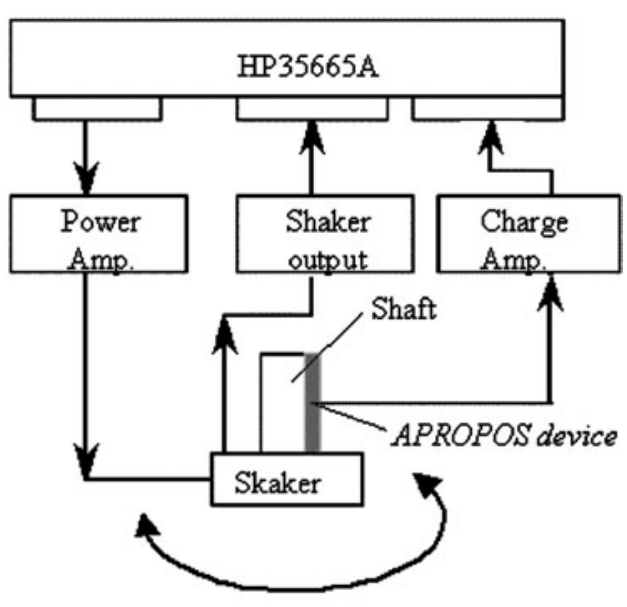

Figure 5. Experimental set-up of an APROPOS device.
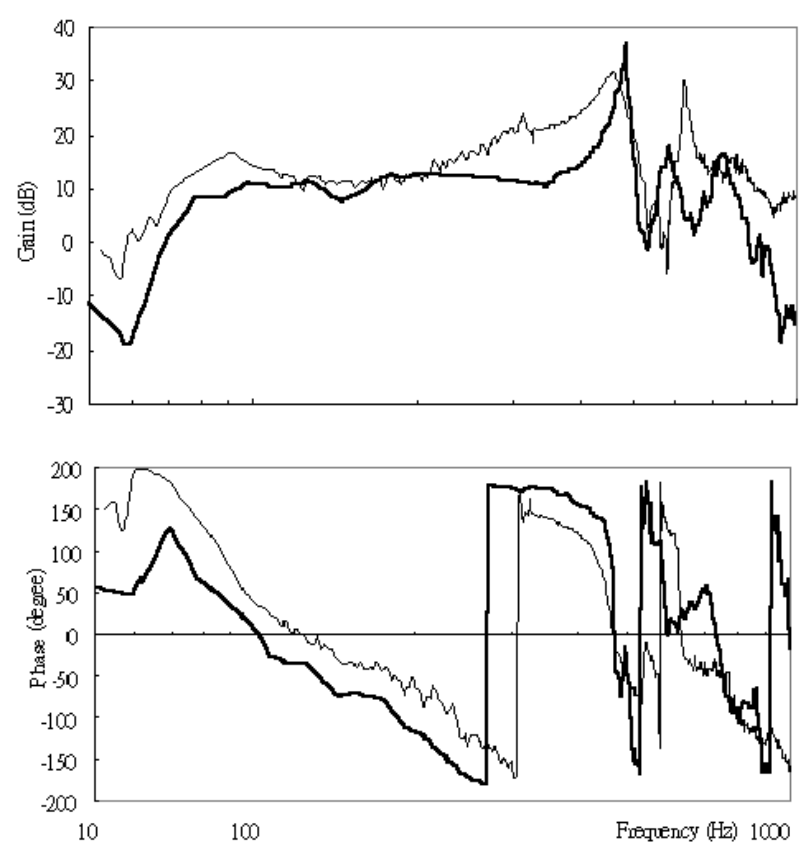

Figure 6. Transfer function of a rotational accelerometer based on an APROPOS device (thick curve) and uniform sensor (thin curve) design.

The corner frequency $\gamma$ of the sine function was chosen to be $95 \mathrm{~Hz}$. Following the above-mentioned APROPOS device design methodology, the corner frequencies $\alpha$ and $\beta$ were chosen to be 200 and $250 \mathrm{~Hz}$ respectively so as to reduce the second mode effect and thus expand the bandwidth. The designed sensor profile and its sensor transfer function are shown in figure 7 and as the thick curves in figure 6 respectively. It is obvious from figure 6 that the introduction of the $-60 \mathrm{~dB} /$ decade low-pass filter from the APROPOS device flattens the bandwidth of the rotational sensor. It should be mentioned that the experimental result of the uniform sensor added a $43 \mathrm{~dB}$ dc gain to its transfer function numerically in figure 6 as compared with the one from the APROPOS device. This shows that a dc gain was introduced, and a $90^{\circ}$ phase shift was also introduced by the odd function APROPOS device. Take this induced $90^{\circ}$ phase shift into 


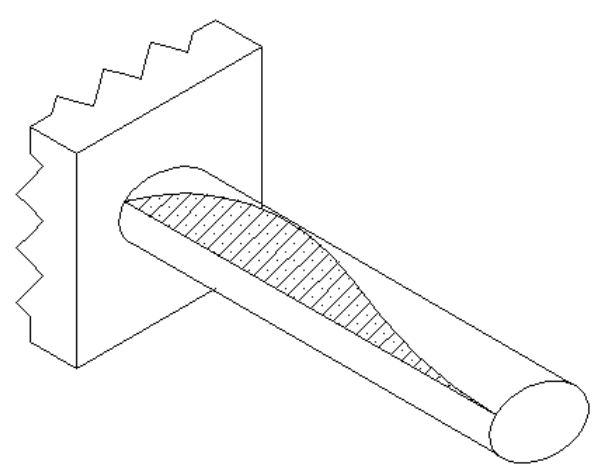

Figure 7. Schematic of a rotational APROPOS device based accelerometer.
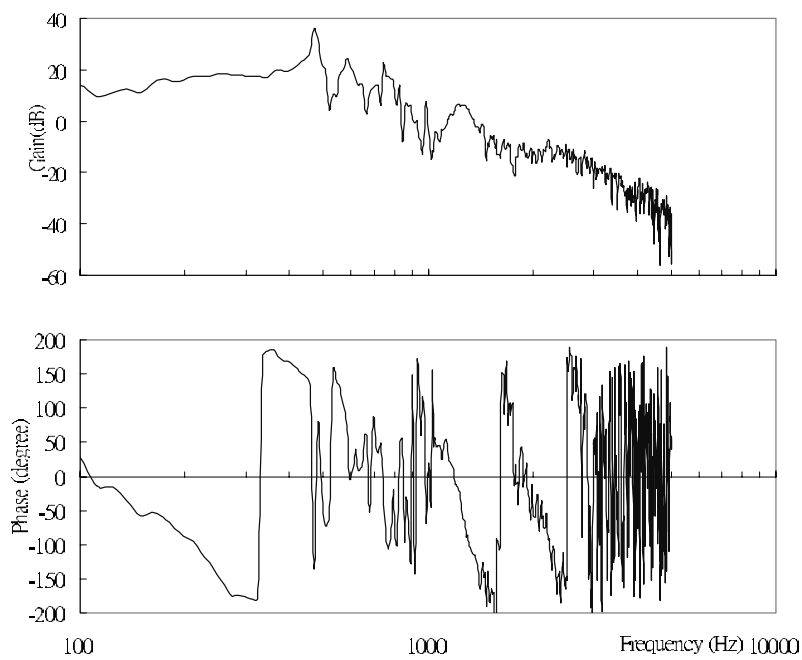

Figure 8. Transfer function of an APROPOS device.

consideration, the phase distribution of the uniform sensor and the APROPOS device shown in figure 6 were identical as predicted by the theory. Considering the frequency range of the corner frequency in 200 and $250 \mathrm{~Hz}$, no additional phase information was introduced into the sensor transfer function. This proved that the APROPOS device was able to perform as a no-phase-delay low-pass filter in spatial domain for a structure in pure rotational vibration. The $60 \mathrm{~dB} /$ decade gain tailoring without phase delay behavior can be observed more clearly in figure 8 . It is clear that the experimental data agrees well with that of the design specifications mentioned above.

\section{Conclusions}

The concept of an APROPOS device for a second-order PDE system was successfully verified by using a one-dimensional shaft. It was shown that by properly weighting all the structure wave modes in an infinite domain mapped from the finite domain using the method of image and window functions, a no-phase-delay low-pass filter can be integrated into the sensor transfer function. It was verified that introduction of the APROPOS device concept can enhance the usable bandwidth of rotational sensors. It should be mentioned again that the performance of a rotational APROPOS devicebased accelerometer is the result of superposition of the characteristics of the structure itself, of the local performance of the targeted origin, and of the extra no-phase-delay filtering introduced by the APROPOS device. To verify the wideband performance of the rotational sensor, the methodology adopted to create a wideband rotational shaker was detailed. It was shown that taking a matching filter to initiate a lowfrequency driving signal to the servomotor and a highfrequency driving signal to the piezoelectric stack could indeed create a wideband rotational shaker with a flat frequency response. In summary, the theory and the design of an experimental set-up for a rotational APROPOS device based accelerometer was successfully verified both theoretically and experimentally.

\section{Acknowledgments}

The authors would like to thank the following for continuous and generous financial support of this research: the National Science Council of Taiwan, ROC, and AHEAD Optoelectronics, Inc. through grants NSC 85-2622-E-002017R, NSC 86-2622-E-002-023R, NSC 88-2218-E-002-005, and NSC 88-2622-E-002-001. The authors would like to express their appreciation to Measurement Specialties, Sensor Products Division (MSI) for continuously providing us with all the PVDF films used in fabricating the piezoelectric devices discussed in the research work.

\section{References}

[1] Prak A, Elwenspoek M and Fluitman J H J 1992 Selective mode excitation and detection of micromachined resonators J. Microelectromech. Syst. 1 179-86

[2] Tzou H S and Hollkamp J J 1994 Collected independent modal control with self-sensing orthogonal piezoelectric actuators (theory and experiment) Smart Mater. Struct. 3 277-84

[3] Tzou H S, Zhong J P and Natori M 1993 Sensor mechanics of distributed shell convolving sensors applied to flexible rings Trans. ASME 115 40-6

[4] Preumont A and Francois A 1999 Piezoelectric array sensing for real-time, broad-band sound radiation measurement ICAST '99: Proc. Int. Conf. on Adaptive Structure and Technologies (Paris, France, 1999) pp 37-44

[5] Hsu Y H and Lee C K 2002 Targeted origin placement for the autonomous gain-phase tailoring of piezoelectric sensors Smart Mater. Struct. 11 444-58

[6] Lee C K 1987 Piezoelectric laminates for torsional and bending modal control: theory and experiment $P h D$ Dissertation Cornell University, Ithaca, New York, USA

[7] Miller D W, Hall S R and von Flotow A H 1990 Development of spatially convolving sensors for structural control applications Proc. AIAA/ASME/ASCE/AHS, Proc. Conf. Structures, Structural Dynamics, and Materials (Long Beach, CA, 1990) pp 2283-97

[8] Colloms M 1997 High Performance Loudspeaker (New York: Wiley)

[9] Graff K F 1975 Wave Motion in Elastic Solids (New York: Dover)

[10] Harris F J 1978 On the use of windows for harmonic analysis with the discrete Fourier transform Proc. IEEE 66 51-83

[11] Campbell C K 1998 Surface Acoustic Wave Device for Mobile and Wireless Communications (San Diego, CA: Academic)

[12] Campbell C K 1989 Surface Acoustic Wave Device and Their Signal Processing Applications (Boston, MA: Academic)

[13] Bode H W 1945 Network Analysis and Feedback Amplifier Design (New York: Van Nostrand-Reinhold) 
[14] Cady W G 1946 Piezoelectricity vol 1 (New York: McGraw-Hill) pp 1-8

[15] Lee C K 1990 Theory of laminated piezoelectric plates for the design of distributed sensors/actuators: part 1. Governing equations and reciprocal relationships J. Acoust. Soc. Am. 87 1144-58
[16] Lee C K 1992 Piezoelectric laminates: theory and experiments for distributed sensors and actuators Intelligent Structural Systems (Netherlands: Kluwer Academic) pp 75-167

[17] Oppenheim A V and Schafer R W 1999 Signal and System (Upper Saddle River, NJ: Prentice-Hall) 